\title{
Interval-valued fuzzy spiking neural P systems for fault diagnosis of power transmission networks
}

\author{
Jun Wang a,c,*, Hong Peng ${ }^{\mathrm{b}}$, Wenping Yu ${ }^{\mathrm{a}}$, Jun Ming a , Mario J. Pérez-Jiménez ${ }^{\mathrm{d}}$, Chengyu Tao a , \\ Xiangnian Huang ${ }^{\mathrm{b}}$
}

\footnotetext{
${ }^{a}$ School of Electrical Engineering and Electronic Information, Xihua University, Chengdu, Sichuan, 610039, China

b School of Computer and Software Engineering, Xihua University, Chengdu, Sichuan, 610039, China

${ }^{\mathrm{c}}$ Key Laboratory of Fluid and Power Machinery, Ministry of Education, Xihua University, Chengdu, 610039, China

${ }^{\mathrm{d}}$ Research Group of Natural Computing, University of Seville, Sevilla, 41012, Spain
}

Keywords:

Power transmission networks Fault diagnosis

Fuzzy spiking neural P systems

Interval-valued fuzzy logic

\begin{abstract}
A B S T R A C T
It is a challenge problem how to deal with the uncertainty in fault diagnosis of power systems. To solve the challenge problem, this paper introduces an interval-valued fuzzy spiking neural P system (IVFSNP system), where the interval-valued fuzzy logic is integrated into spiking neural P systems to characterize the uncertainty. Based on the IVFSNP system, a fuzzy reasoning algorithm is presented, and the corresponding fault diagnosis model is developed. IVFSNP system is capable of describing the incomplete and uncertain fault signals from a supervisory control and data acquisition system equipped together with electric power systems. In order to evaluate the availability and effectiveness of the proposed fault diagnosis model, two case studies of fault diagnosis of a transmission network are discussed and analyzed, including complex and multiple fault situations with the incomplete and uncertain status signals. The results of the case studies demonstrate that IVFSNP system can be used to diagnose the faulty sections in power transmission networks accurately and effectively.
\end{abstract}

\section{Introduction}

A power system is composed of many system sections, such as generators, transformers, bus bars, transmission lines, etc. These system sections are protected by the protective system consisted of protective relays (PRs), circuit breakers (CBs) and communication equipments. Power systems have more and more complex system sections due to their scale extension and increasing structure complexity. When the faults occur, dispatchers need to isolate the influenced system sections accurately and take the necessary means to restore the normal power supply as early as possible. The fault diagnosis of power systems is such a task that distinguishes the faulty system elements according to the operating signals of protective relays and circuit breakers. These operating signals are often retrieved from the supervisor control and data acquisition (SCADA) system in a dispatch center system, which provides the data source for fault diagnosis. However, these signals are usually incomplete and uncertain, especially in complex and multiple fault situations. Therefore, fault diagnosis of power systems becomes an important and challenging task in the situation that has the failures of protective devices, multiple faults, and incomplete and uncertain fault messages.
In the recent years, a variety of artificial intelligence approaches have been developed to deal with fault diagnosis problems of power systems, for example, expert systems (ES) (Lee et al., 2000; Huang, 2002), artificial neural networks (ANN) (Tan and Lim, 2004; Cardoso et al., 2004; Thukaram et al., 2005), Petri nets (PN) (Sun et al., 2004; Luo and Kezunovic, 2008; Chen et al., 2015), heuristic optimization techniques (such as GAs and HBMO) (Lin et al., 2010; Huang et al., 2013), fuzzy logic (FL) (Chin, 2003), fuzzy relation (FR) (Cho and Park, 1997; Chen, 2011), fuzzy digraph model (FDM) (Chen, 2012b), causeeffect network (CEN) (Chen et al., 2011), and so on. ES is an approach that is suitable for operating logics of protective relays and circuit breakers as well as the diagnosis experience of operators. However, it has a slow inference speed and poor tolerance ability. Based on the concept of fuzzy membership, FL can characterize the imprecision and uncertainty in fault diagnosis problems of power systems, but it needs to be combined with other approaches. ANN is an adaptive system that changes its structure based on external and internal information that flows through the network during the learning phase, and it has the advantages of good tolerance and strong learning ability. However, the disadvantages of ANN are the need for numerous samples and poor generally interpreting ability. When PN is applied in fault diagnosis

* Corresponding author at: School of Electrical Engineering and Electronic Information, Xihua University, Chengdu, Sichuan, 610039, China.

E-mail address: wj.xhu@hotmail.com (J. Wang). 
problems, it may suffer from bad tolerance and the difficulty to identify false alarm messages. Moreover, heuristic optimization techniques have the weakness of slow diagnosis and fail to diagnose the faults in the case of inaccurate and loss information. Although FL, FR, FDM and CEN possess a certain degree of advantage when they deal with fault diagnosis problems of power systems, their ability for solving fault diagnosis problems still needs to be investigated further.

Spiking neural P systems (SNP systems, in short) are a class of membrane computing models (Ionescu et al., 2006; Păun et al., 2010), abstracted from the neurophysiological behavior that biological neurons send out electronic pulses along the synapses. SNP systems are distributed and parallel computing models, in which neurons work in parallel. In recent years, a various of SNP systems and P systems were proposed (Song et al., 2013, 2014; Zeng et al., 2014; Peng et al., 2017d; Wu et al., 2018; Pan et al., 2017; Song et al., 2017), and they have been applied to solve different real-world problems (Peng et al., 2015a,b, 2016; Wang et al., 2016; Peng et al., 2017a; Zhang et al., 2018; Liu et al., 2017). Among them, fuzzy spiking neural P systems (FSNP systems, in short) as a new kind of variants were developed by Wang et al. (2013) and Peng et al. (2013), and they have been applied to deal with the fault diagnosis problems of power systems, for example, Tu et al. (2014), Wang et al. (2015), Peng et al. (2017c) and Peng et al. (2018). However, how to handle the incompleteness and uncertainty in fault diagnosis problems of different power systems is an interesting challenge problem and is worth to discuss further. Nowadays, interval-valued fuzzy number is more flexible in dealing with uncertainty, so it has been widely used in risk analysis and group multi-criteria decision making (Wei and Chen, 2009; Chen and Sanguansat, 2011; Chen, 2012a; Baležentis and Zeng, 2013). Although interval-valued fuzzy number seems to be very suited to deal with the incompleteness and uncertainty of fault alarm information, there are no relevant literatures in the field of fault diagnosis of power systems.

In order to solve the challenge problem in power transmission networks, by combining interval-valued fuzzy numbers and logic with SNP systems, a new variant is investigated, called interval-valued fuzzy spiking neural P systems (IVFSNP systems, in short), involving the definition, modeling and fuzzy reasoning method. Main contribution of this paper stays on proposing the IVFSNP systems and developing a fuzzy reasoning algorithm and a fault diagnosis model of power transmission networks. The introduction of interval-valued fuzzy numbers is helpful to characterize and handle the incompleteness and uncertainty of fault alarm messages in power transmission networks. The causality between a faulty section and its protective relays and circuit breakers can be easily and visually modeled by IVFSNP systems, and fault sections for power transmission networks can be quickly diagnosed by their parallel computing mechanism.

The remainder of this paper is organized as follows. IVFSN P systems is introduced in Section 2, including the definition, modeling and reasoning methods. Section 3 describes the fault diagnosis problem, and then discusses fault diagnosis model based on IVFSNP systems. Two case studies of power transmission networks are provided in Section 4. Conclusions are finally drawn in Section 5.

\section{Interval-valued fuzzy spiking neural $P$ systems}

\subsection{Interval-valued fuzzy numbers}

Gorzalczany (1987) presented the concept of interval-valued fuzzy sets. Some basic concepts of interval-valued fuzzy numbers and their arithmetic operations are described as follows. An interval-valued fuzzy number $A$ can be defined as

$A=\left[A^{L}, A^{U}\right]=\left[\left(a_{1}^{L}, a_{2}^{L}, a_{3}^{L}, a_{4}^{L} ; w_{A}^{L}\right),\left(a_{1}^{U}, a_{2}^{U}, a_{3}^{U}, a_{4}^{U} ; w_{A}^{U}\right)\right]$

where $A^{L}$ denotes the lower interval-valued fuzzy number, $A^{U}$ denotes the upper interval-valued fuzzy number, and $A^{L} \subset A^{U}$. The lower

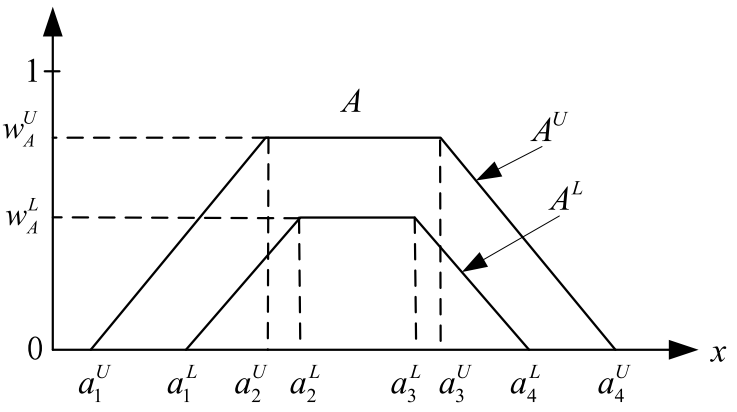

Fig. 1. An interval-valued fuzzy number.

and upper interval-valued fuzzy numbers can be two generalized trapezoidal fuzzy numbers. Fig. 1 shows the membership function curve of the interval-valued fuzzy number $A$, where $a_{1}^{L} \leq a_{2}^{L} \leq a_{3}^{L} \leq a_{4}^{L}, a_{1}^{U} \leq$ $a_{2}^{U} \leq a_{3}^{U} \leq a_{4}^{U}$, and $a_{1}, a_{2}, a_{3}, a_{4}$ are real numbers in $[0,1]$.

Note that if $A^{L}=A^{U}, A$ becomes a generalized fuzzy number; if $a_{1}=a_{2}=a_{3}=a_{4}, A$ becomes a non-negative real number; if $a_{1}<a_{2}=a_{3}<a_{4}, A$ becomes a triangular interval-valued fuzzy number.

Assume that $A=\left[\left(a_{1}^{L}, a_{2}^{L}, a_{3}^{L}, a_{4}^{L} ; w_{A}^{L}\right),\left(a_{1}^{U}, a_{2}^{U}, a_{3}^{U}, a_{4}^{U} ; w_{A}^{U}\right)\right]$ and $B=$ $\left[\left(b_{1}^{L}, b_{2}^{L}, b_{3}^{L}, b_{4}^{L} ; w_{B}^{L}\right),\left(b_{1}^{U}, b_{2}^{U}, b_{3}^{U}, b_{4}^{U} ; w_{B}^{U}\right)\right]$ are two interval-valued fuzzy numbers. Four arithmetic operations for interval-valued fuzzy numbers can be defined as follows:

(1) $A \oplus B=\left[\left(a_{1}^{L}+b_{1}^{L}, a_{2}^{L}+b_{2}^{L}, a_{3}^{L}+b_{3}^{L}, a_{4}^{L}+b_{4}^{L} ; \min \left\{w_{A}^{L}, w_{B}^{L}\right\}\right),\left(a_{1}^{U}+\right.\right.$ $\left.\left.b_{1}^{U}, a_{2}^{U}+b_{2}^{U}, a_{3}^{U}+b_{3}^{U}, a_{4}^{U}+b_{4}^{U} ; \min \left\{w_{A}^{U}, w_{B}^{U}\right\}\right)\right]$.

(2) $A \otimes B=\left[\left(a_{1}^{L} \times b_{1}^{L}, a_{2}^{L} \times b_{2}^{L}, a_{3}^{L} \times b_{3}^{L}, a_{4}^{L} \times b_{4}^{L} ; \min \left\{w_{A}^{L}, w_{B}^{L}\right\}\right),\left(a_{1}^{U} \times\right.\right.$ $\left.\left.b_{1}^{U}, a_{2}^{U} \times b_{2}^{U}, a_{3}^{U} \times b_{3}^{U}, a_{4}^{U} \times b_{4}^{U} \min \left\{w_{A}^{U}, w_{B}^{U}\right\}\right)\right]$.

(3) $A \otimes B=\left[\left(a_{1}^{L} \wedge b_{1}^{L}, a_{2}^{L} \wedge b_{2}^{L}, a_{3}^{L} \wedge b_{3}^{L}, a_{4}^{L} \wedge b_{4}^{L} ; \min \left\{w_{A}^{L}, w_{B}^{L}\right\}\right)\right.$, $\left.\left(a_{1}^{U} \wedge b_{1}^{U}, a_{2}^{U} \wedge b_{2}^{U}, a_{3}^{U} \wedge b_{3}^{U}, a_{4}^{U} \wedge b_{4}^{U} ; \min \left\{w_{A}^{U}, w_{B}^{U}\right\}\right)\right]$.

(4) $A \bigotimes B=\left[\left(a_{1}^{L} \vee b_{1}^{L}, a_{2}^{L} \vee b_{2}^{L}, a_{3}^{L} \vee b_{3}^{L}, a_{4}^{L} \vee b_{4}^{L} ; \min \left\{w_{A}^{L}, w_{B}^{L}\right\}\right)\right.$, $\left.\left(a_{1}^{U} \vee b_{1}^{U}, a_{2}^{U} \vee b_{2}^{U}, a_{3}^{U} \vee b_{3}^{U}, a_{4}^{U} \vee b_{4}^{U} ; \min \left\{w_{A}^{U}, w_{B}^{U}\right\}\right)\right]$.

where for $\forall a, b \in[0,1]$, we define that $a \wedge b=\min \{a, b\}$ and $a \vee b=$ $\max \{a, b\}$.

\subsection{Proposed IVFSNP systems}

The IVFSNP systems are a variant of SNP systems, which integrate interval-valued fuzzy logic into their mechanism. The extension is mainly represented in the following aspects:

(1) The context in each neuron is changed from an integer (the number of spikes) to an interval-valued fuzzy number (the potential value of spikes).

(2) Each neuron is associated with a fuzzy proposition or a fuzzy production rule. For the neuron that describes the fuzzy production rule, an interval-valued fuzzy number $c$ is introduced to express the confidence factor (CF) of the fuzzy production rule.

(3) There is only an extended spiking (firing) rule, of the form $a^{\theta} \rightarrow$ $a^{\beta}$ (or $a^{\theta} \rightarrow a^{\theta}$ ), where $\theta, \beta$ are two interval-valued fuzzy numbers. If a neuron contains a spike with value $\theta$, then its spiking rule is enabled, hence, it fires to generate a spike with value $\beta$ (or $\theta$ ) and the generated spike is sent to its successor neurons.

The proposed IVFSNP systems are given as follows.

Definition 1. An IVFSNP system of degree $m$ is a construct

$\Pi=\left(A, \sigma_{1}, \sigma_{2}, \ldots, \sigma_{m}\right.$, syn $\left., I, O\right)$

where: 


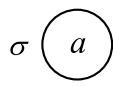

(a)

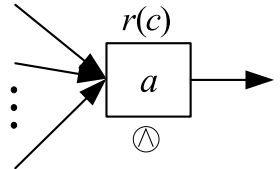

(b)

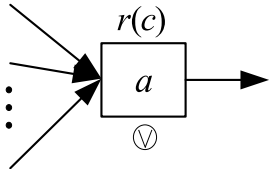

(c)
Fig. 2. (a) a proposition neuron, (b) a $Q$-type rule neuron and (c) a $\emptyset$-type rule neuron.

(1) $A=\{a\}$ is a singleton alphabet ( $a$ is called spike);

(2) $\sigma_{1}, \sigma_{2}, \ldots, \sigma_{m}$ are neurons of the form $\sigma_{i}=\left(\theta_{i}, c_{i}, r_{i}\right)$, $i \in\{1,2, \ldots, m\}$, where:

(a) $\theta_{i}$ is an interval-valued fuzzy number representing the value of spikes initially contained in neuron $\sigma_{i}$;

(b) $c_{i}$ is an interval-valued fuzzy number, which expresses the confidence factor for a fuzzy production rule or is omitted in the case of representing a fuzzy proposition;

(c) $r_{i}$ is a firing rule of neuron $\sigma_{i}$, with the form $a^{\theta} \rightarrow a^{\theta}$ or $a^{\theta} \rightarrow a^{\beta}$, where $\theta$ and $\beta$ are two interval-valued fuzzy numbers.

(3) syn $\subseteq\{1,2, \ldots, m\} \times\{1,2, \ldots, m\}$, with $(i, i) \notin$ syn for $\forall 1 \leq i \leq m$ is a synapse graph, defining the synapses between neurons;

(4) $I$ and $O$ denote the sets of input neurons and output neurons, respectively.

To deal with fuzzy logic, the neurons in IVFSNP systems are further divided into three types: proposition neurons, $₫$-type rule neurons and Q-type rule neurons. The neurons of three types are defined as follows.

Definition 2. Proposition neuron is the neuron that is associated with a fuzzy proposition in a fuzzy knowledge base.

Fig. 2(a) shows a proposition neuron $\sigma$ denoted by a circular symbol, where symbol $a$ denotes the spike contained in the neuron.

Definition 3. Q-type rule neuron is the neuron that is associated with an "and"-type fuzzy production rule in a fuzzy knowledge base, where the confidence factor (CF) of the fuzzy production rule is expressed by $c$.

Fig. 2(b) shows a $\bigotimes$-type rule neuron $\sigma$ denoted by a rectangle symbol with $\oslash$, where symbol $a$ denotes the spike, symbol $r$ denotes a firing rule of the form $a^{\theta} \rightarrow a^{\beta}$, and symbol $c$ is the confidence factor.

Definition 4. (-type rule neuron is the neuron that is associated with an "or"-type fuzzy production rule in a fuzzy knowledge base, where the confidence factor (CF) of the fuzzy production rule is expressed by $c$.

Fig. 2(c) shows a $\emptyset$-type rule neuron $\sigma$ denoted by a rectangle symbol with $\emptyset$, where symbols $a, r$ and "c" are semantically the same as that in Fig. 2(b).

\subsection{Modeling fuzzy production rules}

The fuzzy production rules have been commonly used in knowledge representation, where "and" and "or" operations are used to connect multiple propositions respectively. There are two main types of fuzzy production rules:

Type 1: if $p_{1}$ and $p_{2}$ and $\ldots$ and $p_{k-1}$ then $p_{k}(\mathrm{CF}=c)$

Type 2: if $p_{1}$ or $p_{2}$ or $\ldots$ or $p_{k-1}$ then $p_{k}(\mathrm{CF}=c)$ where $p_{1}, p_{2}, \ldots, p_{k-1}, p_{k}$ are $k$ propositions, and $c$ is an interval-valued fuzzy number used to denote the confidence factor $(\mathrm{CF})$ of the fuzzy production rule.

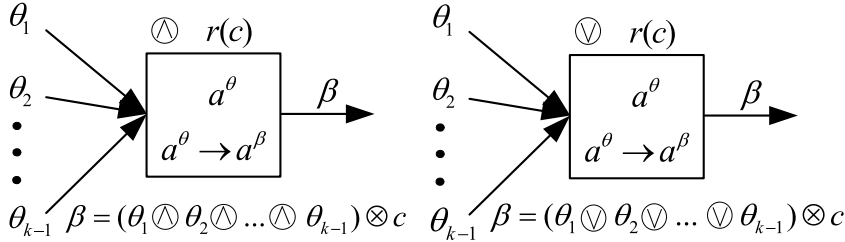

(a)

(b)

Fig. 3. Modeling (a) type 1-fuzzy production rule and (b) type 2-fuzzy production rule using IVFSN P systems, respectively.

A fuzzy production rule of type 1 can be denoted by a $₫$-type rule neuron, shown in Fig. 3(a). The $k-1$ antecedent propositions of the rule correspond to $k-1$ proposition neurons, while the consequent proposition is associated with a proposition neuron. If the $\bigotimes$-type rule neuron receives $k-1$ spikes with values $\theta_{1}, \theta_{2}, \ldots, \theta_{k-1}$ from its antecedent proposition neurons, then the spike value of the rule neuron is $\theta=\theta_{1} \otimes \theta_{2} \otimes \cdots \otimes \theta_{k-1}$. Once the rule neuron fires, it can emit a spike with value $\beta=\theta \otimes c$ to the consequent proposition neuron. Thus, $\beta=\left(\theta_{1} \otimes \theta_{2} \otimes \cdots \otimes \theta_{k-1}\right) \otimes c$.

Similarly, a fuzzy production rule of type 2 can be denoted by a $\emptyset$ type rule neuron, shown in Fig. 3(b). If the $\bigotimes$-type rule neuron receives $k-1$ spikes with values $\theta_{1}, \theta_{2}, \ldots, \theta_{k-1}$ from the antecedent proposition neurons, then the spike value is $\theta=\theta_{1} \otimes \theta_{2} \oslash \cdots \otimes \theta_{k-1}$. When the rule neuron fires, it can emit a spike with value $\beta=\theta \otimes c$ to the consequent proposition neuron. Thus, $\beta=\left(\theta_{1} \oslash \theta_{2} \oslash \cdots \otimes \theta_{k-1}\right) \otimes c$.

\subsection{Fuzzy Reasoning algorithm}

A fuzzy reasoning algorithm based on IVSNP systems is introduced. Suppose that the considered IVFSNP system has $m$ proposition neurons and $n$ rule neurons that are one of $\bigotimes$-type and $\bigotimes$-type rule neurons, hence the total number of neurons is $s=m+n$. To explain the presented fuzzy reasoning algorithm, some vectors and matrices are described as follows.

(1) $\theta=\left(\theta_{1}, \theta_{2}, \ldots, \theta_{m}\right)^{T}$ is a vector combined by the fuzzy values of $m$ proposition neurons. If a proposition neuron has no spike, its pulse value is given as "unknown" or $[(0.0,0.0,0.0,0.0 ; 0.8),(0.0$, $0.0,0.0,0.0 ; 1.0)]$.

(2) $\delta=\left(\delta_{1}, \delta_{2}, \ldots, \delta_{n}\right)^{T}$ is a vector containing the fuzzy values of $n$ rule neurons. If a rule neuron has no spike, its pulse value is given as "unknown" or $[(0.0,0.0,0.0,0.0 ; 0.8),(0.0,0.0,0.0,0.0 ; 1.0)]$.

(3) $C=\operatorname{diag}\left(c_{1}, c_{2}, \ldots, c_{n}\right)$ is a diagonal matrix consisting of the certainty factors of $n$ fuzzy production rules.

(4) $D_{1}=\left(d_{i j}\right)_{m \times n}$ is a matrix representing the synaptic connections from proposition neurons to $\oslash$-type rule neurons. If there is a synapse connection from proposition neuron $\sigma_{i}$ to rule neuron $\sigma_{j}, d_{i j}=1$; otherwise, $d_{i j}=0$.

(5) $D_{2}=\left(d_{i j}\right)_{m \times n}$ is a matrix representing the synaptic connections from proposition neurons to $\bigotimes$-type rule neurons. If there is a synapse connection from proposition neuron $\sigma_{i}$ to rule neuron $\sigma_{j}, d_{i j}=1$; otherwise, $d_{i j}=0$.

(6) $E=\left(e_{j i}\right)_{n \times m}$ is a matrix representing the synaptic connections from rule neurons to proposition neurons. If there is a synapse connection from rule neuron $\sigma_{j}$ to proposition neuron $\sigma_{i}, e_{j i}=1$; otherwise, $e_{j i}=0$.

Moreover, three multiplication operations are given as follows.

(1) $C \odot \delta=\left(c_{1} \otimes \delta_{1}, c_{2} \otimes \delta_{2}, \ldots, c_{n} \otimes \delta_{n}\right)^{T}$.

(2) $D^{T} \odot \theta=\left(\bar{d}_{1}, \bar{d}_{2}, \ldots, \bar{d}_{n}\right)^{T}$, where $\bar{d}_{j}=d_{1 j} \theta_{1} \oslash d_{2 j} \theta_{2} \oslash \ldots \otimes d_{m j} \theta_{m}$, $j=1,2, \ldots, n$.

(3) $E^{T} \circledast \delta=\left(\bar{e}_{1}, \bar{e}_{2}, \ldots, \bar{e}_{m}\right)^{T}$, where $\bar{e}_{i}=e_{1 i} \delta_{1} \oslash e_{2 i} \delta_{2} \oslash \ldots \emptyset e_{n i} \delta_{n}$, $j=1,2, \ldots, m$. 
Table 1

The proposed fuzzy reasoning algorithm.

Input: $D_{1}, D_{2}, E, C$, and $O_{1}=$ (unknown, .., unknown) (halting condition)

Output: $\left\{\theta_{t j} \mid \sigma_{j} \in O\right\}$

Begin

(1) $\theta_{0} \leftarrow\left(\theta_{10}, \theta_{20}, \ldots, \theta_{m 0}\right)$

(2) $\delta_{0} \leftarrow\left(\delta_{10}, \delta_{20}, \ldots, \delta_{n 0}\right)$;

(3) $t \leftarrow 1$;

(4) while $\left(\delta_{t} \neq O_{1}\right)$

(5) $\quad \delta_{t} \leftarrow\left(D_{1}^{T} \odot \theta_{t-1}\right) \oplus\left(D_{2}^{T} \circledast \theta_{t-1}\right)$;

(6) $\quad \theta_{t} \leftarrow\left(E^{T} \circledast\left(C \odot \delta_{t}\right)\right)$;

(7) $\quad t \leftarrow t+1$;

(8) end while

End

Table 2

Linguistic values and the corresponding interval-valued fuzzy numbers.

\begin{tabular}{ll}
\hline Linguistic terms & Interval-valued fuzzy number (IVFN) \\
\hline Absolutely-low(AL) & {$[(0.0,0.0,0.0,0.0 ; 0.8),(0.0,0.0,0.0,0.0 ; 1.0)]$} \\
Very-low(VL) & {$[(0.0075,0.0075,0.015,0.0525 ; 0.8),(0.0,0.0,0.02,0.07 ; 1.0)]$} \\
Low(L) & {$[(0.0875,0.12,0.16,0.1825 ; 0.8),(0.04,0.10,0.18,0.23 ; 1.0)]$} \\
Fairly-low(FL) & {$[(0.2325,0.255,0.325,0.3575 ; 0.8),(0.17,0.22,0.36,0.42 ; 1.0)]$} \\
Medium(M) & {$[(0.4025,0.4525,0.5375,0.5675 ; 0.8),(0.32,0.41,0.58,0.65 ; 1.0)]$} \\
Fairly-high(FH) & {$[(0.65,0.6725,0.7575,0.79 ; 0.8),(0.58,0.63,0.80,0.86 ; 1.0)]$} \\
High(H) & {$[(0.7825,0.815,0.885,0.9075 ; 0.8),(0.72,0.78,0.92,0.97 ; 1.0)]$} \\
Very-high(VH) & {$[(0.9475,0.985,0.9925,0.9925 ; 0.8),(0.93,0.98,1.0,1.0 ; 1.0)]$} \\
Absolutely-high(AH) & {$[(1.0,1.0,1.0,1.0 ; 1.0),(1.0,1.0,1.0,1.0 ; 1.0)]$} \\
\hline
\end{tabular}

Based on the firing mechanism of IVSNP systems, the proposed fuzzy reasoning algorithm can be described in Table 1 . Note that the inputs of the system are the fuzzy values of the propositions associated with the input proposition neurons, and when the reasoning is completed, its outputs are the fuzzy values of the propositions associated with the output proposition neurons.

\section{Fault diagnosis model based on IVFSNP systems}

\subsection{Problem description}

The fault diagnosis of power transmission systems is a process of identifying faulty components by using the tripping signals of protection relays and circuit breakers (CBs). Assume that these tripping signals are from a SCADA system equipped with power transmission systems. In order to quickly and selectively remove the faulty components, main measure is a three-section current protection, i.e., main protection, nearby (first) backup protection and remote (second) backup protection, when a fault occurs in power transmission network. In this paper, two types of fault diagnosis are mainly considered: lines and buses.

Fig. 4 shows a $345 \mathrm{kV}$ power transmission system. This system consists of 18 system sections, 17 circuit breakers and 60 protective relays. For the convenience of description, some notations are described as follows. A bus, line and $\mathrm{CB}$ are denoted by $B U S, L$ and $C B$, respectively. The 18 system sections are labeled as $B U S 18, B U S 19$, $\ldots, B U S 25, B U S 27$ and $L 23, L 24, \ldots, L 31$. The $17 \mathrm{CBs}$ are labeled as $C B 45, \ldots, C B 60$ and $C B 62$. The 60 protective relays are composed of 26 main relays ( $B U S 18 m, \ldots, B U S 25 m, B U S 27 m, L 23-x m, \ldots$, $L 31-x m), 17$ nearby backup relays $(L 23-x b, \ldots, L 31-x b)$, and 17 remote backup relays $(L 23-x s, \ldots, L 31-x s)$.

The operational principle of protective relays of power transmission networks is described as follows.

(1) Protective relays of lines: When a fault occurs on a line, main protective relays (MPRs) of the line operate, and the corresponding CBs are tripped. For example, if line $L 24$ fails, MPRs $L 24-$ $18 m$ and $L 24-20 m$ operate to trip CB47 and CB48. Similarly, when main protections of this line fail to operate, nearby backup protective relays (NBPRs) operate to trip the CBs connected to this line. For example, if line $L 24$ fails and MPR $L 24-18 m$ fails to operate, NBPR $L 24-18 b$ operates to trip CB47. If line $L 24$ fails and MPR $L 24-20 m$ fails to operate, NBPR $L 24-20 b$ operates to trip $C B 48$. In addition, when a section in adjacent region of a line fails and its protections fail to operate, the remote protective relays (RBPRs) of the line operate to protect the section. For example, if section $B U S 18$ fails and $C B 47$ fails to trip off, RBPR $L 24-18$ s operates to trip $C B 47$. If section $B U S 20$ fails and $C B 48$ fails to trip off, RBPR $L 24-20$ s operates to trip $C B 48$.

(2) Protective relays of buses: When MPRs of a bus operate, all CBs directly connected to the bus will be tripped. For instance, if bus $B U S 22$ fails, MPR $B 22 m$ operates to trip $C B 51$. Note that there are not any NBPRs for buses. Therefore, when MPRs of a bus fail to operate, RBPRs of all the adjacent regions, which can protect the bus, operate to trip off the relevant CBs. For example, if bus $B U S 22$ fails and MPR B22m fails to operate, RBPR $L 26-22 s$ operates to trip $C B 51$ so that the bus can be protected.

\subsection{Fault diagnosis model}

In this study, IVFSNP systems are used to realize fault diagnosis of main components (lines and buses) in power systems, in particular, for the case with incomplete and uncertain signals of protective devices and CBs. The diagnosis process of IVFSNP systems is described as follows. Firstly, a fault diagnosis model for each suspicious component in power transmission system is built by an IVFSNP system. Secondly, the operational signals of protective devices are retrieved from the SCADA system; Next, each fault diagnosis model uses the proposed fuzzy reasoning algorithm to obtain fault confidence levels of suspicious faulty components. Finally, each faulty component is identified according to its fault confidence level.

The causality between a fault and its protective devices can be expressed by some fault fuzzy production rules for main components including lines and buses in power transmission systems. Each fault fuzzy production rule ha a certainty factor, which represents the degree of confidence that a fault occurs. Considering the uncertainty in the knowledge of experts and power dispatchers, linguistic terms are used to describe the certainty factors, which are denoted by interval-valued fuzzy numbers and provided in Table 2.

Based on the experience and the levels of protection, some provisions in used in this work can be illustrated as follows. Firstly, the certainty factors of rule neurons related to both main protections and nearby backup protections are set to be $\mathrm{AH}$; the certainty factors of rule neurons related to remote backup protections are set to be $\mathrm{VH}$; if it involves multiple levels of protections, the certainty factor is set to be the value corresponding to the highest level of protections. Secondly, if the confidence level $\theta$ of a section satisfies the condition $\theta \geq$ $[(0.65,0.6725,0.7575,0.79 ; 0.8),(0.58,0.63,0.80,0.86 ; 1.0)]$, the section is identified as a fault; if $\theta \leq[(0.2325,0.255,0.325,0.3575 ; 0.8),(0.17,0.22$, $0.36,0.42 ; 1.0)]$, the section is not faulty; otherwise, the section may be faulty.

In addition, the status signals retrieved from the SCADA system may include operation failure, mal-operation and misinformation, so it is necessary to use a confidence level to characterize the operation accuracy of each section. Therefore, an empirical confidence level is assigned to each protective device including the protective relays and its corresponding CBs. Tables 3 and 4 list the confidence levels of the operated protective devices and the non-operated protective devices used in this work, respectively.

In practice, a large-scale power transmission system consists of many system sections. Each system section will correspond to an IVFSNP subsystem. Thus, fault diagnosis model of the power transmission system consists of a lot of IVFSNP subsystems. Note that These IVFSNP subsystems are parallel. Therefore, fault diagnosis model based on IVFSNP systems can realize a fast computation. 


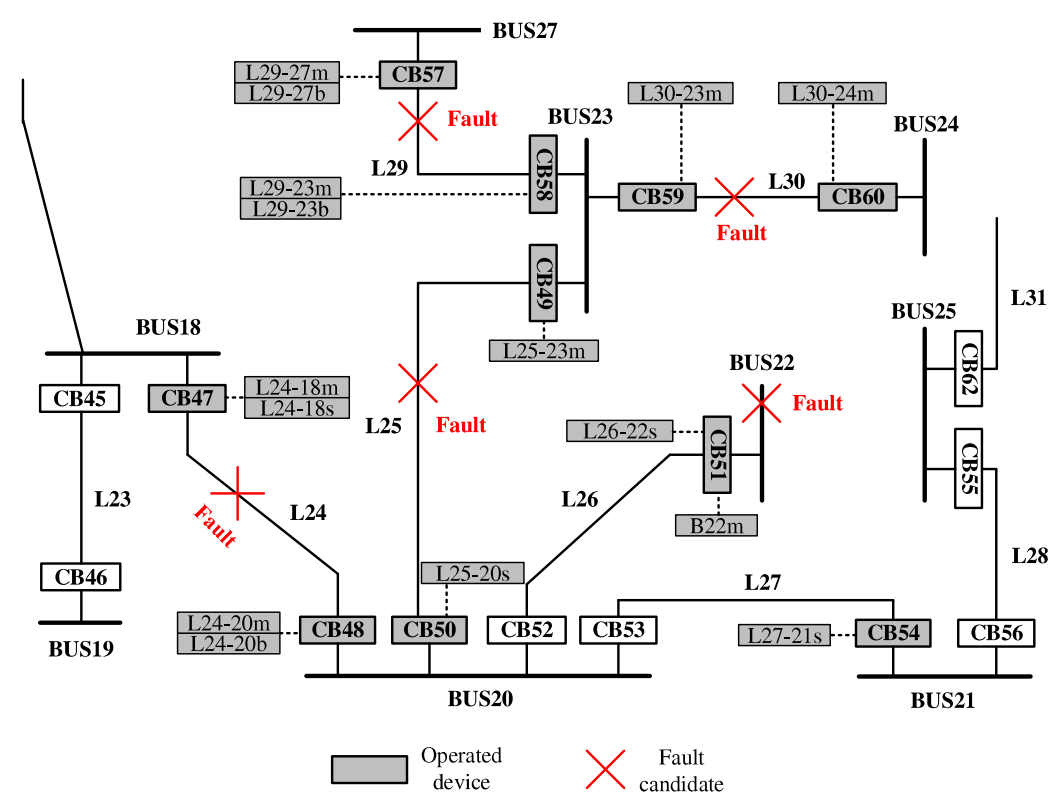

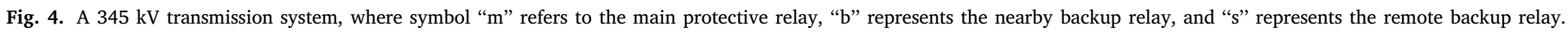

Table 3

Confidence levels of the operated protective devices.

\begin{tabular}{|c|c|c|c|c|c|c|}
\hline \multirow[t]{3}{*}{ Sections } & \multicolumn{6}{|c|}{ Protective devices } \\
\hline & \multicolumn{2}{|l|}{ Main } & \multicolumn{2}{|l|}{ Nearby } & \multicolumn{2}{|c|}{ Remote } \\
\hline & Relays & CBs & Relays & CBs & Relays & CBs \\
\hline $\mathrm{L}$ & $\mathrm{VH}$ & VH & $\mathrm{H}$ & $\mathrm{H}$ & FH & $\mathrm{FH}$ \\
\hline B & VH & VH & - & - & FH & FH \\
\hline
\end{tabular}

Table 4

Confidence levels of the non-operated protective devices.

\begin{tabular}{|c|c|c|c|c|c|c|}
\hline \multirow[t]{3}{*}{ Sections } & \multicolumn{6}{|c|}{ Protective devices } \\
\hline & \multicolumn{2}{|l|}{ Main } & \multicolumn{2}{|l|}{ Nearby } & \multicolumn{2}{|c|}{ Remote } \\
\hline & Relays & $\overline{\mathrm{CBs}}$ & Relays & $\overline{\mathrm{CBs}}$ & Relays & $\mathrm{CBs}$ \\
\hline $\mathrm{L}$ & $\mathrm{L}$ & $\mathrm{L}$ & $\mathrm{L}$ & $\mathrm{L}$ & $\mathrm{L}$ & $\mathrm{L}$ \\
\hline B & FL & $\mathrm{L}$ & - & - & FL & $\mathrm{L}$ \\
\hline
\end{tabular}

\section{Case studies}

In this section, two case studies of a typical system in power systems are analyzed to demonstrate the effectiveness and superiority of the proposed IVFSNP systems: a $345 \mathrm{kV}$ transmission system. These cases include complex faults and multiple faults with the incomplete and uncertain status information. The diagnosis results of IVFSNP systems in the same cases are compared with other diagnosis methods.

\subsection{Illustration example: $345 \mathrm{kV}$ transmission system}

This example is used to discuss the complex faults and multiple faults with rejection and incorrect and uncertain information. A $345 \mathrm{kV}$ power transmission system is studied, shown in Fig. 4, and two cases are discussed.

\subsubsection{Case 1 (complex faults with failure devices and incorrect signals)}

Suppose that complex faults occur at transmission lines $L 29$ and $L 30$, as shown in Fig. 4. For line section L29, its MPRs $L 29-27 m$ and $L 29-23 m$ operate to trip off the corresponding CBs CB57 and CB58, but they fail. Thus, the NFPRs corresponding to line $L 29, L 29-27 b$ and $L 29-23 b$, operate to trip off the CBs again. Status information

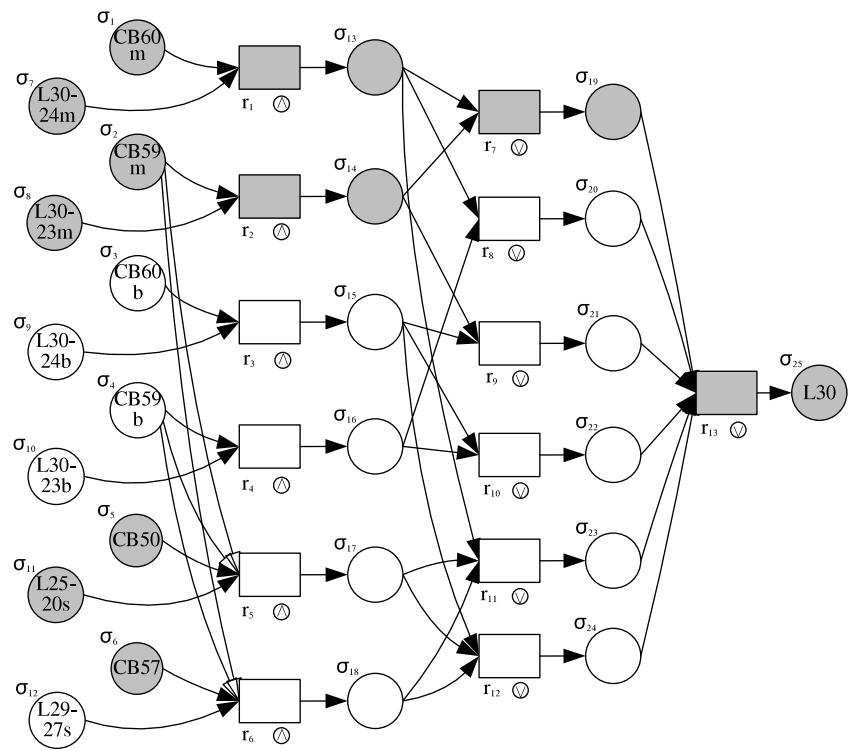

Fig. 5. The fault diagnosis model of line $L 30$ based on IVFSNP systems.

obtained from the SCADA system are as follows: the operated relays are $L 30-23 m, L 30-24 m, L 29-27 m, L 29-23 m, L 29-27 b, L 29-23 b$ and $L 25-20 s$, and the tripped CBs have CB50,CB57, CB58,CB59 and CB60.

Firstly, the fault diagnosis models of lines $L 29$ and L30, which each include 25 proposition neurons and 13 rule neurons, are constructed based on IVFSNP systems. Fig. 5 shows the fault diagnosis model of line $L 30$. However, the fault diagnosis model of line $L 29$ is not provided due to space limitation.

Secondly, since $L 29$ and $L 30$ have a similar reasoning process, the fuzzy reasoning process of $L 30$ as an example is illustrated as follows. Initially, interval-valued fuzzy numbers $\theta_{0}$ and $\delta_{0}$ can be obtained according to the status information of Case 1 and Tables 2-4. In the case study, $\theta$ is a 25 dimensional vector, and $\delta$ is a 13 dimensional 
vector.

$[(0.9475,0.985,0.9925,0.9925 ; 0.8),(0.93,0.98,1.0,1.0 ; 1.0)]$ $[(0.9475,0.985,0.9925,0.9925 ; 0.8),(0.93,0.98,1.0,1.0 ; 1.0)]$ $[(0.0875,0.12,0.16,0.1825 ; 0.8),(0.04,0.10,0.18,0.23 ; 1.0)]$ $[(0.0875,0.12,0.16,0.1825 ; 0.8),(0.04,0.10,0.18,0.23 ; 1.0)]$ $[(0.0,0.0,0.0,0.0 ; 0.8),(0.0,0.0,0.0,0.0 ; 1.0)]$ $[(0.0,0.0,0.0,0.0 ; 0.8),(0.0,0.0,0.0,0.0 ; 1.0)]$

$\theta_{0}=$ $[(0.9475,0.985,0.9925,0.9925 ; 0.8),(0.93,0.98,1.0,1.0 ; 1.0)]$ $[(0.9475,0.985,0.9925,0.9925 ; 0.8),(0.93,0.98,1.0,1.0 ; 1.0)]$ $[(0.0875,0.12,0.16,0.1825 ; 0.8),(0.04,0.10,0.18,0.23 ; 1.0)]$ $[(0.0875,0.12,0.16,0.1825 ; 0.8),(0.04,0.10,0.18,0.23 ; 1.0)]$ $[(0.0,0.0,0.0,0.0 ; 0.8),(0.0,0.0,0.0,0.0 ; 1.0)]$ $[(0.0,0.0,0.0,0.0 ; 0.8),(0.0,0.0,0.0,0.0 ; 1.0)]$ $O$

$\delta_{0}=[O]$;

When $g=1$, we get the results

$\delta_{1}=\left[\begin{array}{c}{[(0.9475,0.985,0.9925,0.9925 ; 0.8),(0.93,0.98,1.0,1.0 ; 1.0)]} \\ {[(0.9475,0.985,0.9925,0.9925 ; 0.8),(0.93,0.98,1.0,1.0 ; 1.0)]} \\ {[(0.0875,0.12,0.16,0.1825 ; 0.8),(0.04,0.10,0.18,0.23 ; 1.0)]} \\ {[(0.0875,0.12,0.16,0.1825 ; 0.8),(0.04,0.10,0.18,0.23 ; 1.0)]} \\ O\end{array}\right]$

$\theta_{1}=\left[\begin{array}{c}O \\ {[(0.9475,0.985,0.9925,0.9925 ; 0.8),(0.93,0.98,1.0,1.0 ; 1.0)]} \\ {[(0.9475,0.985,0.9925,0.9925 ; 0.8),(0.93,0.98,1.0,1.0 ; 1.0)]} \\ {[(0.0875,0.12,0.16,0.1825 ; 0.8),(0.04,0.10,0.18,0.23 ; 1.0)]} \\ {[(0.0875,0.12,0.16,0.1825 ; 0.8),(0.04,0.10,0.18,0.23 ; 1.0)]} \\ O\end{array}\right] ;$

When $g=2$, we get the results

$\delta_{2}=\left[\begin{array}{c}O \\ {[(0.9475,0.985,0.9925,0.9925 ; 0.8),(0.93,0.98,1.0,1.0 ; 1.0)]} \\ {[(0.9475,0.985,0.9925,0.9925 ; 0.8),(0.93,0.98,1.0,1.0 ; 1.0)]} \\ {[(0.9475,0.985,0.9925,0.9925 ; 0.8),(0.93,0.98,1.0,1.0 ; 1.0)]} \\ {[(0.0875,0.12,0.16,0.1825 ; 0.8),(0.04,0.10,0.18,0.23 ; 1.0)]} \\ {[(0.9475,0.985,0.9925,0.9925 ; 0.8),(0.93,0.98,1.0,1.0)]} \\ {[(0.0875,0.12,0.16,0.1825 ; 0.8),(0.04,0.10,0.18,0.23 ; 1.0)]} \\ {[(0.0,0.0,0.0,0.0 ; 0.8),(0.0,0.0,0.0,0.0 ; 1.0)]}\end{array}\right]$

$\theta_{2}=\left[\begin{array}{c}O \\ {[(0.9475,0.985,0.9925,0.9925 ; 0.8),(0.93,0.98,1.0,1.0 ; 1.0)]} \\ {[(0.9475,0.985,0.9925,0.9925 ; 0.8),(0.93,0.98,1.0,1.0 ; 1.0)]} \\ {[(0.9475,0.985,0.9925,0.9925 ; 0.8),(0.93,0.98,1.0,1.0)]} \\ {[(0.0875,0.12,0.16,0.1825 ; 0.8),(0.04,0.10,0.18,0.23 ; 1.0)]} \\ {[(0.9475,0.985,0.9925,0.9925 ; 0.8),(0.93,0.98,1.0,1.0 ; 1.0)]} \\ {[(0.0875,0.12,0.16,0.1825 ; 0.8),(0.04,0.10,0.18,0.23 ; 1.0)]} \\ {[(0.0,0.0,0.0,0.0 ; 0.8),(0.0,0.0,0.0,0.0 ; 1.0)]}\end{array}\right] ;$

When $g=3$, we get the results

$\delta_{3}=\left[\begin{array}{c}O \\ {[(0.9475,0.985,0.9925,0.9925 ; 0.8),(0.93,0.98,1.0,1.0 ; 1.0)]}\end{array}\right]$

$\theta_{3}=\left[\begin{array}{c}O \\ {[(0.9475,0.985,0.9925,0.9925 ; 0.8),(0.93,0.98,1.0,1.0 ; 1.0)]}\end{array}\right]$;

When $g=4$, we get the result

$\delta_{4}=[O]$.

Thus, the termination condition is satisfied and the reasoning process ends. The reasoning result is $[(0.9475,0.985,0.9925,0.9925 ; 0.8)$, $(0.93,0.98,1.0,1.0 ; 1.0)]$ from output neuron $\sigma_{25}$. According to the judgment condition in Section 3.2, L30 is a faulty section with a confidence level VH. Similarly, we can also obtain that the confidence level of $L 25$ is $[(0.0875,0.12,0.16,0.1825 ; 0.8)$

, $(0.04,0.10,0.18,0.23 ; 1.0)]$. Since the confidence level of $L 25$ is $\mathrm{L}, L 25$ is not a faulty section. Therefore, $L 25-20 s$ is an incorrect trapping signal.

Likewise, the reasoning result of $L 29$ is $[(0.7825,0.815,0.885$, $0.9075 ; 0.8),(0.72,0.78,0.92,0.97 ; 1.0)]$ from output neuron $\sigma_{25}$. According to the judgment condition in Section 3.2, L29 is a faulty section with a confidence level $\mathrm{H}$. In this situation, the rejection of CBs CB57

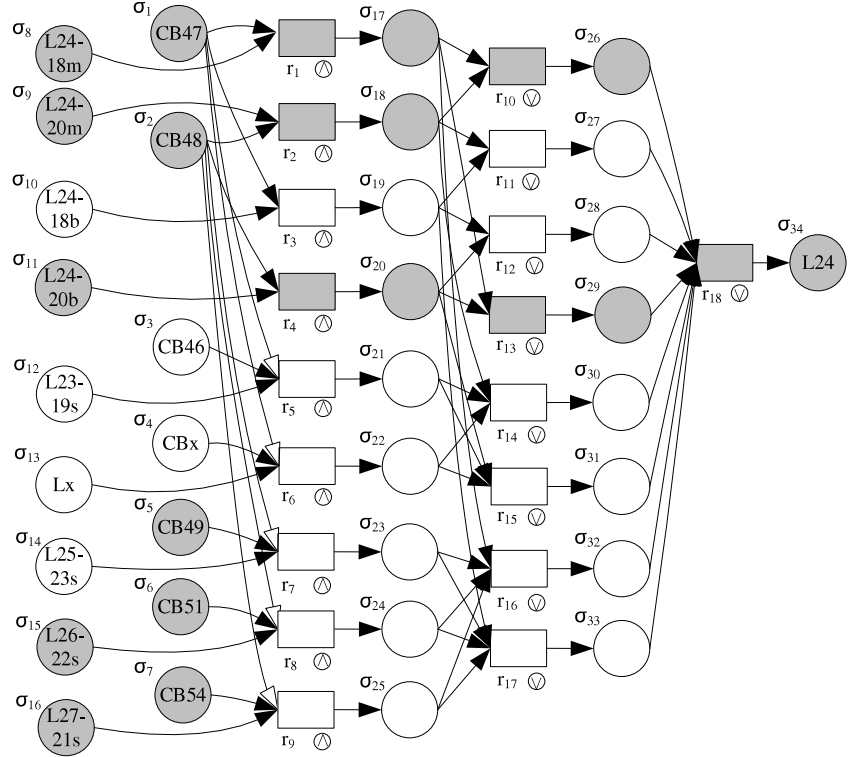

Fig. 6. The fault diagnosis model of line $L 24$ based on IVFSNP systems.

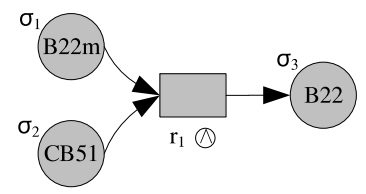

Fig. 7. The fault diagnosis model of line $B 22$ based on IVFSNP systems.

and $C B 58$ after the MPRs $L 29-27 m$ and $L 29-23 m$ operate results in the failure diffusion.

The results of Case 1 illustrate that for complex fault problem with failure devices and incorrect signals, IVFSNP systems not only can distinguish whether some section is a faulty section with a certain confidence level, but also determine the incorrect signals.

\subsubsection{Case 2 (multiple faults with uncertain information)}

The following status information is obtained from the SCADA system: the operated relays are $L 24-18 m, L 24-18 s, L 24-20 m, L 24-20 b$, $L 25-23 m, L 26-22 s, L 27-21 s$ and $B 22 m$, and the tripped CBs have $C B 47, C B 48, C B 49, C B 51$ and $C B 54$. It can be seen from the obtained information that there are two possible information with the faults: one is that the operating of $L 24-20 \mathrm{~m}$ and the rejection of $C B 48$ result in the operating of $L 24-20 b$ and the trapping of $C B 48$ again; another is that $L 24-20 b$ is an incorrect tripping signal.

The fault diagnosis models of lines $L 24$ and $L 25$, which each include 34 proposition neurons and 18 rule neurons, are constructed using two IVFSNP systems respectively. Due to space limitation, fault diagnosis model of line $L 24$ is shown in Fig. 6, however, that of line $L 25$ is omitted here. Fig. 7 gives the fault diagnosis models of lines $B 22$ based on IVFSNP systems, consisting of 3 proposition neurons and a rule neuron.

Since the fuzzy reasoning process of Case 2 is similar to Case 1 , it is omitted here. After reasoning, we can obtain that lines $L 24, L 25$ and bus $B 22$ have the same confidence level, [ $(0.9475,0.985,0.9925,0.9925 ; 0.8)$, $(0.93,0.98,1.0,1.0 ; 1.0)]$. According to the judgment condition in Section 3.2, $L 24, L 25$ and $B 22$ all are identified as the faulty sections with the confidence level VH. Similarly, we can also obtain that the confidence levels of lines $L 26$ and $L 27$ are [ $(0.0875,0.12,0.16,0.1825 ; 0.8)$, $(0.04,0.10,0.18,0.23 ; 1.0)]$. The confidence levels of $L 25$ and $L 27$ are $L$, so they both are not the faulty sections. 
Table 5

Comparisons between IVFSNP systems and other three fault diagnosis methods.

\begin{tabular}{lllll}
\hline Cases & \multicolumn{2}{l}{ Diagnosis results } & & \\
\cline { 2 - 5 } & IVFSNP systems & FL (Chin, 2003) & FR (Cho and Park, 1997) & FDM (Chen, 2012b) \\
\hline 1 & L29, L30 & L30 & L30 & L29, L30 \\
2 & B22, L24, L25 & B22 & B22 & - \\
\hline
\end{tabular}

\subsection{Comparison analysis with other methods}

The $345 \mathrm{kV}$ transmission system has been discussed in a number of literatures, fox example, fuzzy logic (Chin, 2003), fuzzy relation (Cho and Park, 1997) and fuzzy digraph model (FDM) (Chen, 2012b). The example is used to compare the abilities of IVFSNP systems and other three diagnosis methods on diagnosing complex faults and multiple faults with rejection and incorrect and uncertain information. The comparison results of the four diagnosis methods in cases 1 and 2 are provided in Table 5 .

\subsubsection{Comparison analysis on Case 1}

The diagnosis results of IVFSNP systems are the same to that of FDM (Chen, 2012b): $L 30$ and $L 29$ are recognized as the faulty sections. However, for $L 25$, the confidence level obtained by IVFSNP systems is L, while the confidence level obtained by FDM is 0.3 . Therefore, compared with FDM, IVFSNP systems not only can identify that $L 25$ is not a faulty section with higher confidence level, but also can judge that $L 25-20$ s is an incorrect tripping signal.

The results in literature (Chin, 2003) showed that FL method can diagnose only a faulty section $L 30$ and a non-faulty section $L 25$. However, L29 is not recognized as a faulty section because an error occurs. The error is caused by some incorrect signals.

In literature Cho and Park (1997), the membership degrees of L30 and $L 29$ obtained by FR method are 0.998 and 0.509 respectively. Therefore, $L 30$ is distinguished as a faulty section because of its high membership degree. However, $L 29$ is not identified as a faulty section because of its lower membership degree and an error existed in the line. The error is caused by the incorrect operation of $L 25-20$ s associated with $L 30$.

In summary, IVFSNP systems can distinguish all faulty sections, even if there are the incorrect signals. Therefore, IVFSNP systems are suitable to deal with complex fault problems with failure devices and incorrect signals.

\subsubsection{Comparison analysis on Case 2}

In this paper, lines $L 24, L 25$ and $B 22$ are distinguished as the faulty sections with the confidence level VH. But, lines $L 26$ and $L 27$ are identified as the non-faulty sections because of their lower confidence levels. Note that Case 3 was not considered in FDM (Chen, 2012b).

In literature (Chin, 2003), it can be distinguished by FL method that $B 22$ is a faulty section and $L 26, L 27$ are the non-faulty sections. However, FL method cannot identify whether L24 and L25 are the faulty sections because an error occurs.

The membership degree of $B 22$ obtained by FR method (Cho and Park, 1997) is 0.937 , so it is distinguished as a faulty section. The membership degrees of $L 24$ and $L 25$ are 0.673 and 0.792 , respectively. Since there is the rejection device in the line and the MPRs in lines $L 24$ and $L 25$ do not operate, $L 24$ and $L 25$ are identified as the faulty sections even if they have higher membership degrees.

The comparison demonstrates that IVFSNP systems can effectively deal with multiple fault problems with uncertain information. In addition, the fault confidence levels represented by interval-valued fuzzy numbers provide a quantitative description of the fault components and make the diagnosis results more reliable. Owing to linguistic terms including uncertainty in certain extent are more flexible than probability values, the interval-valued fuzzy numbers corresponding to the linguistic terms provide a more intuitive way for experts and dispatchers to understand the diagnosis results.

\section{Conclusions}

In this paper, IVFSNP systems as a novel graphic modeling tool are proposed to diagnose main faulty sections in power transmission networks. This approach provides a good diagnosis solution due to its graphical modeling ability and intelligent reasoning process, and it also provides a more convenient way for readers to understand the process and results of fault diagnosis in power transmission systems. IVFSNP systems have a fast diagnosis process due to their distributed parallel computing feature. Compared with conventional fuzzy numbers, interval-valued fuzzy numbers contain more uncertainty and can better reflect fuzziness of subjective judgment. Therefore, IVFSNP systems can handle incomplete and uncertain tripping signals from the SCADA system in a more flexible and effective way by using interval-valued fuzzy numbers, which adequately displays the fault-tolerant capacity of this proposed method. The case studies on power transmission networks demonstrate that the proposed diagnosis method can effectively and accurately deal with complex faults and multiple faults with protection devices and CBs failure, incorrect and uncertain tripping signals.

\section{Acknowledgments}

This work was partially supported by the Research Fund of Sichuan Science and Technology Project (No. 2018JY0083), Chunhui Project Foundation of the Education Department of China (Nos. Z2016143 and Z2016148), and Research Foundation of the Education Department of Sichuan province (No. 17TD0034), China.

\section{References}

Baležentis, T., Zeng, S.Z., 2013. Group multi-criteria decision making based upon interval-valued fuzzy numbers: an extension of the multimoora method. Expert Syst. Appl. 40 (2), 543-550.

Cardoso, G., Rolim, J.G., Zürn, H.H., 2004. Application of neural-network modules to electric power system fault section estimation. IEEE Trans. Power Deliv. 19 (3), 1034-1041.

Chen, W.H., 2011. Fault section estimation using fuzzy matrix-based reasoning methods. IEEE Trans. Power Deliv. 26 (1), 205-213.

Chen, T.Y., 2012a. Multiple criteria group decision-making with generalized intervalvalued fuzzy numbers based on signed distances and incomplete weights. Appl. Math. Mod. 36 (7), 3029-3052.

Chen, W.H., 2012b. Online fault diagnosis for power transmission networks using fuzzy digraph models. IEEE Trans. Power Deliv. 27 (2), 688-698.

Chen, S.M., Sanguansat, K., 2011. Analyzing fuzzy risk based on similarity measures between interval-valued fuzzy numbers. Expert Syst. Appl. 38 (7), 8612-8621.

Chen, W.H., Tsai, S.H., Liu, H.I., 2011. Fault section estimation for power networks using logic cause-effect models. IEEE Trans. Power Deliv. 26 (2), 963-971.

Chen, S.J., Zhan, T.S., Huang, C.H., Chen, J.L., Lin, C.H., 2015. Nontechnical loss and outage detection using fraction-order self-synchronization error-based fuzzy petri nets in micro-distribution systems. IEEE Trans. Smart Grid 6 (1), 411-420.

Chin, H.C., 2003. Fault section diagnosis of power system using fuzzy logic. IEEE Trans. Power Syst. 18 (1), 245-250.

Cho, H.J., Park, J.K., 1997. An expert system for fault section diagnosis of power systems using fuzzy relations. IEEE Trans. Power Syst. 12 (1), 342-348.

Gorzalczany, M.B., 1987. A method of inference in approximate reasoning based on interval-valued fuzzy sets. Fuzzy Sets and Systems 21 (1), 1-17.

Huang, Y.C., 2002. Fault section estimation in power systems suing novel decision support systems. IEEE Trans. Power Syst. 17 (2), 439-444.

Huang, S.J., Liu, X.Z., Su, W.F., Ou, T.C., 2013. Application of honey-bee mating optimization algorithm to fault section estimation in power systems. IEEE Trans. Power Deliv. 28 (3), 1944-1951.

Ionescu, M., Păun, Gh., Yokomori, T., 2006. Spiking neural p systems. Fund. Inf. 71, 279-308.

Lee, H.J., Ahn, B.S., Park, Y.M., 2000. A fault diagnosis expert system for distribution substations. IEEE Trans. Power Deliv. 15 (1), 92-97. 
Lin, X.N., Ke, S.H., Li, Z.T., Weng, H.L., Han, X.H., 2010. A fault diagnosis method of power systems based on improved objective function and genetic algorithm-tabu search. IEEE Trans. Power Deliv. 25 (3), 1268-1274.

Liu, X., Zhao, Y., Sun, M., 2017. An improved apriori algorithm based on an evolutioncommunication tissue-like p system with promoters and inhibitors. Discrete Dyn. Nat. Soc. 2017, 6978146, 1-11.

Luo, X., Kezunovic, M., 2008. Implementing fuzzy reasoning Petri-nets for fault section estimation. IEEE Trans. Power Deliv. 23 (2), 676-685.

Pan, L., Gh. Păun, Zhang, G., Neri, F., 2017. Spiking neural p systems with communication on request. Int. J. Neural Syst. 28 (8), 1750042, 1-13.

Peng, H., Shi, P., Wang, J., Riscos-Núñez, A., Pérez-Jiménez, M.J., 2017a. Multiobjective fuzzy clustering approach based on tissue-like membrane systems. Knowl.-Based Syst. $125,74-82$.

Peng, H., Wang, J., Ming, J., Shi, P., Pérez-Jiménez, M.J., Yu, W., Tao, C., 2018. Fault diagnosis of power systems using intuitionistic fuzzy spiking neural P systems. IEEE Trans. Smart Grid 9 (5), 4777-4784.

Peng, H., Wang, J., Pérez-Jiménez, M.J., Riscos-Núñez, A., 2015a. An unsupervised learning algorithm for membrane computing. Inform. Sci. 304 (20), 80-91.

Peng, H., Wang, J., Pérez-Jiménez, M.J., Wang, H., Shao, J., Wang, T., 2013. Fuzzy reasoning spiking neural p system for fault diagnosis. Inform. Sci. 235 (20), 106-116.

Peng, H., Wang, J., Shi, P., Pérez-Jiménez, M.J., Riscos-Núñez, A., 2016. An extended membrane system with active membrane to solve automatic fuzzy clustering problems. Int. J. Neural Syst. 26, 1-17.

Peng, H., Wang, J., Shi, P., Pérez-Jiménez, M.J., Riscos-Núñez, A., 2017c. Fault diagnosis of power systems using fuzzy tissue-like P systems. Integr. Comput.-Aided Eng. 24, 401-411.

Peng, H., Wang, J., Shi, P., Riscos-Núñez, A., Pérez-Jiménez, M.J., 2015b. An automatic clustering algorithm inspired by membrane computing. Pattern Recognit. Lett. 68 (15), 34-40.

Peng, H., Yang, J., Wang, J., Wang, T., Sun, Z., Song, X., Luo, X., Huang, X., 2017d. Spiking neural p systems with multiple channels. Neural Netw. 95, 66-71.
Pǎun, Gh., Rozenberg, G., Salomaa, A., 2010. The Oxford HandBook of Membrance Computing. New York: Oxford University Press.

Song, T., Pan, L.Q., Păun, Gh., 2013. Asynchronous spiking neural p systems with local synchronization. Inform. Sci. 219 (10), 197-207.

Song, T., Pan, L., Păun, G., 2014. Spiking neural p systems with rules on synapses. Theoret. Comput. Sci. 529, 82-95.

Song, B., Zhang, C., Pan, L., 2017. Tissue-like P systems with evolutional symport/antiport rules. Inform. Sci. 378, 177-193.

Sun, J., Qin, S.Y., Song, Y.H., 2004. Fault diagnosis of electric power systems based on fuzzy Petri nets. IEEE Trans. Power Syst. 19 (4), 2053-2059.

Tan, S.C., Lim, C.P., 2004. Application of an adaptive neural network with synbolic rule extraction to fault detection and diagnosis in a power generation plnt. IEEE Trans. Energy Conversion 19 (2), 369-377.

Thukaram, D., Khincha, H.P., Vijaynarasimha, H.P., 2005. Artificial neural network and support vector machine approach for locating faults in radial distribution systems. IEEE Trans. Power Deliv. 20 (2), 710-721.

Tu, M., Wang, J., Peng, H., Shi, P., 2014. Application of adaptive fuzzy spiking neural P systems in fault diagnosis of power systems. Chin. J. Elect. 23 (1), 87-92.

Wang, J., Shi, P., Peng, H., 2016. Membrane computing model for IIR filter design. Inform. Sci. 329 (1), 164-176.

Wang, J., Shi, P., Peng, H., Pérez-Jiménez, M.J., Wang, T., 2013. Weighted fuzzy spiking neural P system. IEEE Trans. Fuzzy Syst. 21 (2), 209-220.

Wang, T., Zhang, G.X., Zhao, J.B., He, Z.Y., Wang, J., Pérez-Jiménez, M.J., 2015. Fault diagnosis of electric power systems based on fuzzy reasoning spiking neural $\mathrm{P}$ systems. IEEE Trans. Power Syst. 30 (3), 1182-1194.

Wei, S.H., Chen, S.M., 2009. Fuzzy risk analysis based on interval-valued fuzzy numbers. Expert Syst. Appl. 36 (2), 2285-2299.

Wu, T., Păun, A., Zhang, Z., Pan, L., 2018. Spiking neural p systems with polarizations. IEEE Trans. Neural Netw. Learn. Syst. 29 (8), 3349-3360.

Zeng, X.X., Zhang, X.Y., Song, T., Pan, L.Q., 2014. Spiking neural p systems with thresholds. Neural Comput. 26 (7), 1340-1361.

Zhang, Z., Wu, T., Păun, A., Pan, L., 2018. Universal enzymatic numerical P systems with small number of enzymatic variables. Sci. China: Inf. Sci. 61 (9), 092103. 